\title{
A Shielding Concept for the MedAustron Facility
}

\author{
L. Jägerhofer*1 ${ }^{1}$, E. Feldbaumer ${ }^{2}$, S. Roesler ${ }^{2}$, C. Theis ${ }^{2}$ and H. Vincke ${ }^{2}$
}

\author{
${ }^{I}$ EBG MedAustron GmbH, Austria \\ *Lukas.Jaegerhofer@medaustron.at \\ ${ }^{2}$ CERN, HSE-RP, Geneva 23, CH-1211 Geneva, Switzerland
}

\begin{abstract}
MedAustron is a synchrotron based accelerator facility for cancer therapy and research in Wiener Neustadt, $50 \mathrm{~km}$ south of Vienna. The facility will provide protons up to kinetic energies of $250 \mathrm{MeV}$ and carbon ions up to $400 \mathrm{MeV} / \mathrm{n}$ for ion beam therapy. Additionally, protons up to $800 \mathrm{MeV}$ kinetic energy will be used in a dedicated room for non-clinical research. In order to obtain a shielding concept for this facility a detailed geometry of the accelerator facility was implemented into the Monte-Carlo code FLUKA and shielding simulations were performed. In the course of these simulations the contributions of different particle types to the mixed fields around the accelerator and behind shielding were analysed. In an iterative process with the architect the final design of the shielding concept was developed until it was capable of reducing the effect of secondary radiation on humans and the environment below Austrian legal limits.
\end{abstract}

\section{KEYWORDS: EBG MedAustron GmbH, Shielding Calculations, Hadron Therapy, FLUKA}

\section{Introduction}

MedAustron is a synchrotron based accelerator facility which was built in Wiener Neustadt, Austria. It will provide proton beams up to kinetic energies of $250 \mathrm{MeV}$ and ion beams up to $400 \mathrm{MeV} / \mathrm{n}$ for cancer treatment. It is planned to treat 1200 patients per year as an ambulatory. Apart from clinical treatment, the facility will provide one target room with up to $800 \mathrm{MeV}$ protons for non-clinical research in the fields of medical radiation physics, radiation biology and experimental physics. The medical application has defined the accelerator design for MedAustron but from a radiation protection point of view the protons with $800 \mathrm{MeV}$ play an important role. Figure 1 shows the patients' entrance of the facility.

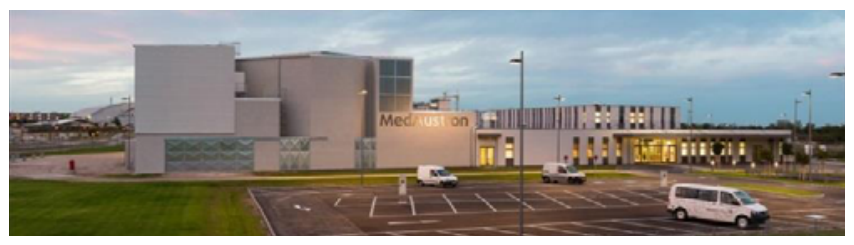

Figure 1. Patients'entrance of the MedAustron facility (1)

Beam operation in the MedAustron facility produces secondary radiation fields due to beam losses along the accelerator chain and the beam transfer line as well as due to intended irradiation of objects or persons in the treatment rooms. Therefore it is required to shield the facility sufficiently. The Monte Carlo Code FLUKA (2) (3) was used to determine the shielding concept which reduces the effect of this secondary radiation on humans and environment below acceptable values, according to the legal limits in Austria.
In order to account for uncertainties of the Monte-Carlo simulations and the fact that incorporation of radionuclides is contributing to the accumulated effective dose, all simulation results are evaluated with a safety factor of 10 . This results in dose limits of $0.1 \mathrm{mSv}$ in publicly accessible areas, $0.6 \mathrm{mSv}$ for supervised areas and $2 \mathrm{mSv}$ for controlled areas which are accessible during operation. It has to be mentioned that the whole process of shielding design was iterative. The wall thicknesses were adapted to our demands, according to the simulation results. Then the geometry was adapted and simulations were repeated until the dose map met the requirements of legal dose limits outside as well as inside radiation areas.

\section{The MedAustron Accelerator Facility}

\section{1 lon beam therapy}

MedAustron offers an innovative form of radiation therapy (ion beam therapy) using protons and carbon ions. Compared to conventional radiation therapy, this treatment is able to reduce radiation exposure to adjacent healthy tissue and to spare the tissue behind the tumour almost entirely. Therefore, ion beam therapy is an optimal treatment for tumours close to radiosensitive organs, like the brain and the spinal cord, eyes, liver and lungs. Since tissue in growth is more sensitive to radiation, proton radiation is particularly suitable for cancer in children and adolescents. The focus lies on reducing the risk of long-term consequences by minimising dose to healthy tissue. (4)

\subsection{Irradiation rooms}

MedAustron has four irradiation rooms. Three will be used 
for patient treatment and one for non-clinical research. Though the rooms differ in terms of flexibility of the particle beam, they all have one thing in common: each of the four rooms is equipped with an innovative robotic positioning system. This will allow direct comparisons and applications of the results drawn from non-clinical research for the patient treatment. (4)

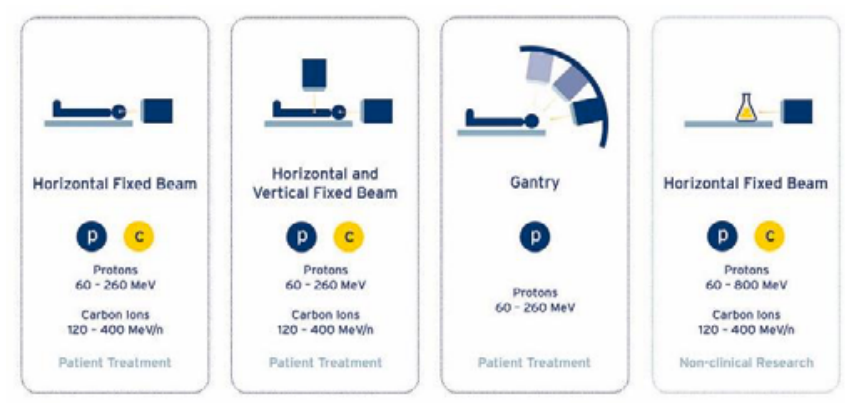

Figure 2. Four different irradiation rooms (4)

\subsection{Base parameters of the MedAustron beams}

Although the medical applications defined the accelerator design, the beam parameters for the experimental operation and the medical operation are different. Table 1 shows possible beam parameters for protons and carbon ions.

Table 1. Beam parameters at extraction from the synchrotron.

\begin{tabular}{|l|l|}
\hline Protons & \\
Kinetic energy (min. - max.) & $60-800 \mathrm{MeV}$ \\
Beam intensity & $\leq 2 \times 10^{10}$ particles per pulse \\
Extraction time (min. - max.) & $0.1-10 \mathrm{~s}$ \\
Repetition rate (max.) & $1 \mathrm{~Hz}$ \\
\hline Carbon ions & \\
Kinetic energy (min. - max.) & $120-400 \mathrm{MeV} / \mathrm{n}$ \\
Beam intensity & $\leq 1 \times 10^{9} \mathrm{particles}$ per pulse \\
Extraction time (min. - max.) & $0.1-10 \mathrm{~s}$ \\
Repetition rate (max.) & $1 \mathrm{~Hz}$ \\
\hline
\end{tabular}

\section{Monte Carlo simulations}

\subsection{Setup of the simulation geometry}

To assess the necessary shielding, simulations with the Monte-Carlo code FLUKA were carried out. For the verification of sufficient shielding a three-dimensional geometry was developed and implemented in FLUKA. Figure 3 shows a three dimensional view of the geometry visualized in SimpleGeo. (5) Ceilings are not shown in this picture for better understanding. The interior and exterior of this model are filled with air. Simplified models of dipoles and quadrupoles are placed in crucial positions to take the shielding effect of their massive iron yokes into account. The emergency exits, the air ducts and some representative cable ducts are included in the model.

Along the beam line numerous places can be defined where particles will be lost due to beam optic devices like dumps or magnets. Also at the end of the beam line in each of the 4 treatment rooms particles are delivered according to irradiation plans. All these particles contribute to the annual ambient dose equivalent in that area and build the basis for the simulation of a detailed dose map. Due to the fact that the contribution of the particles to the ambient dose equivalent is strongly related to the energy of the particles, only losses with maximum energies of $250 \mathrm{MeV}$ and $800 \mathrm{MeV}$ for protons and $400 \mathrm{MeV} / \mathrm{n}$ for carbon ions were taken into account for the simulations.

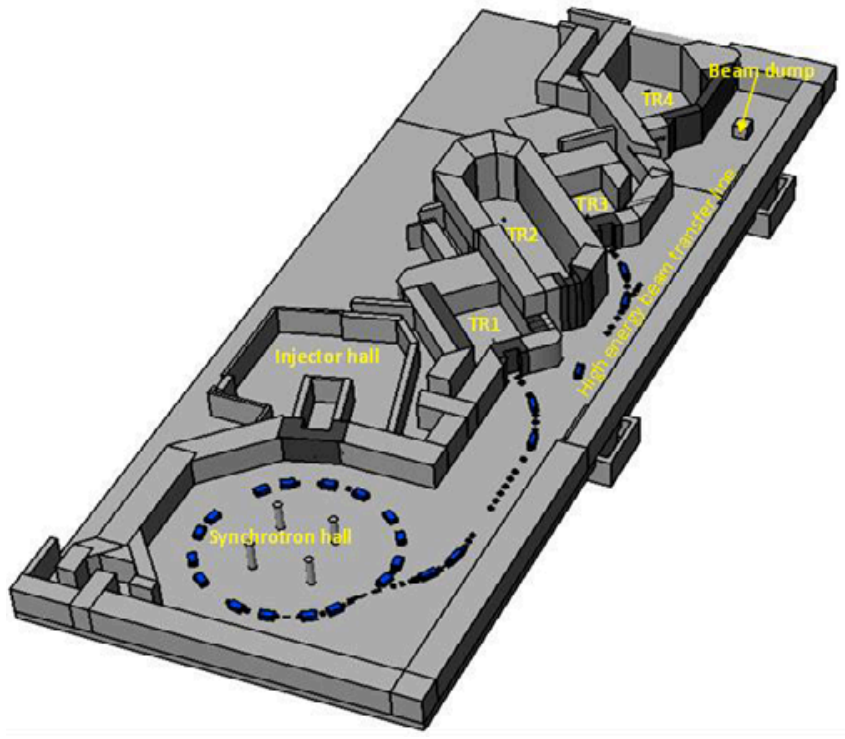

Figure 3. Schematic view of the 3-D FLUKA model of the MedAustron accelerator complex.

\subsection{Method for the reduction of variance}

To reduce simulation time significantly biasing methods were introduced. These mathematical methods are used in Monte Carlo simulations to reduce the variance of the results in relevant areas behind thick shielding. For the following simulations those areas, which are adjacent to the room during treatment, are considered to be relevant and may be accessed. A so called "importance biasing" method was applied. With this method importance factors are assigned to different regions to increase the statistical weight of each particle entering the region. For this purpose all walls and the ceilings as well were divided in $40 \mathrm{~cm}$ slabs, which is approximately the inelastic scattering length of the high energy cascade caused by 800 $\mathrm{MeV}$ protons in the MedAustron concrete. These sectors (40 cm slabs) were then assigned an importance factor from the inside to the outside according to the formula

importancefactor $=$ startvalue $* e^{\text {number of sector }}$

Figure 4 shows irradiation room 1 as an example for importance biasing. The air in the maze has to be biased to assure increasing importance factors along the paths of each particle. By these means statistical uncertainties below $20 \%$ could be obtained at relevant places outside of the shielding. 


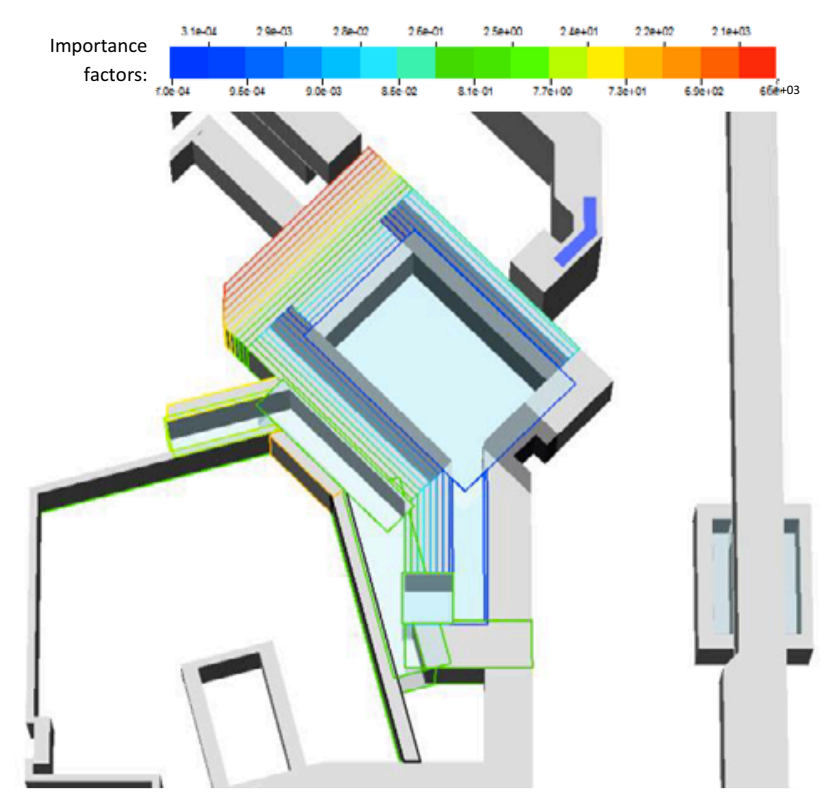

Figure 4. Importance biasing for irradiation room 1. The colours represent the importance factors.

\subsection{Results of the Monte Carlo Simulations}
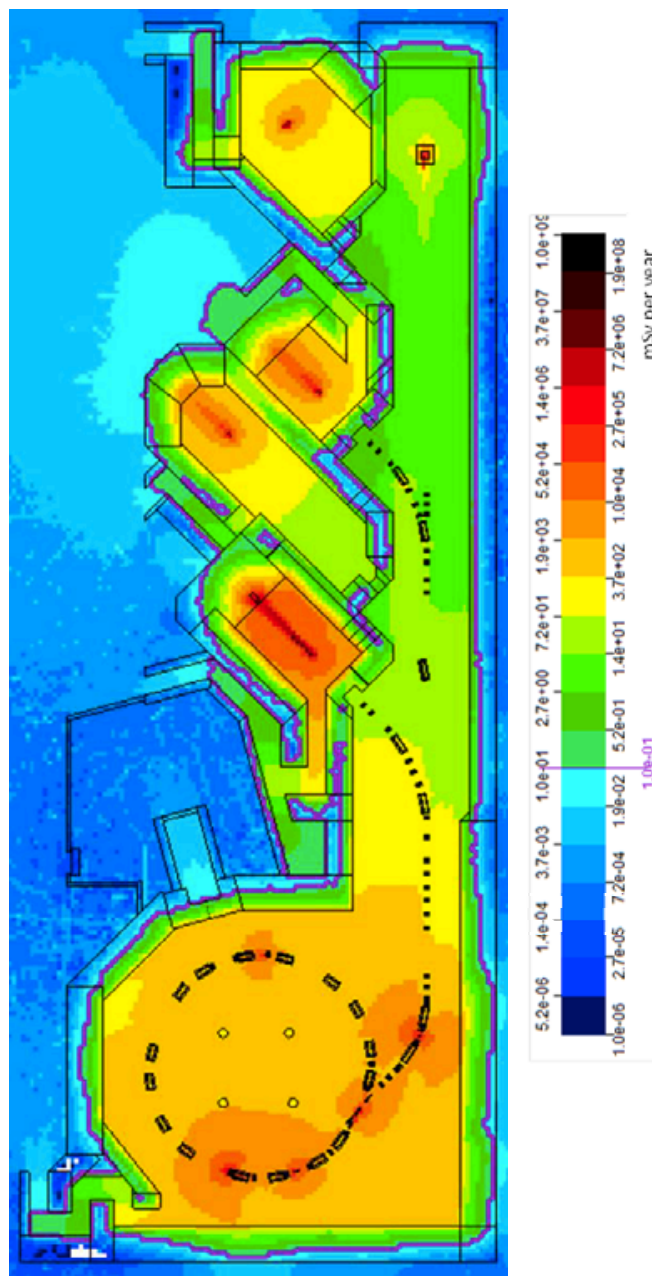

Figure 5. Annual ambient dose equivalent map of the full MedAustron facility at beam height.
A full annual ambient dose equivalent map of the MedAustron accelerator facility as well as close surroundings can be obtained by summation of the ambient dose equivalent originating from each simulated loss points and the treatment rooms. The resulting dose map is shown in Figure 5 and provides a clear indication that the shielding concept is capable of reducing the effect of secondary radiation on humans and the environment below Austrian legal limits. The purple line indicates the self-introduced $0.1 \mathrm{mSv}$ limit.

\subsection{Analysis of the radiation environment}

Considering that different types of particles in mixed radiation fields contribute differently to the total ambient dose equivalent rates inside and around the MedAustron facility at neuralgic points, it is of great interest to quantify these contributions accurately. Furthermore, for the conception of a detector system it is crucial to know the composition of the radiation environment at the position of the detector to be able to install an appropriate detector and furthermore make correct measurements of ambient dose equivalent rates.

\subsubsection{Spectra originating from the main loss points in the Synchrotron hall}

To obtain the spectra at the positions shown in Figure 6 particle losses of the $800 \mathrm{MeV}$ protons at the main loss points in the Synchrotron hall, the horizontal beam dump, the vertical beam dump, the electrostatic extraction septum and the magnetic extraction septum were summed up and weighted accordingly to the respective annual particle losses at each loss point. The following spectra represent only particle losses of $800 \mathrm{MeV}$ protons, since these beams as well as their secondary radiation represent the main contribution to the annual ambient dose equivalent rate inside and outside of shielding at MedAustron. The spectra are scored inside air filled boxes of the dimensions $100 \times 100 \times 200 \mathrm{~cm}^{3}$ standing at the floor level. Scoring volumes are distributed over the whole facility, here the synchrotron is chosen as an example.

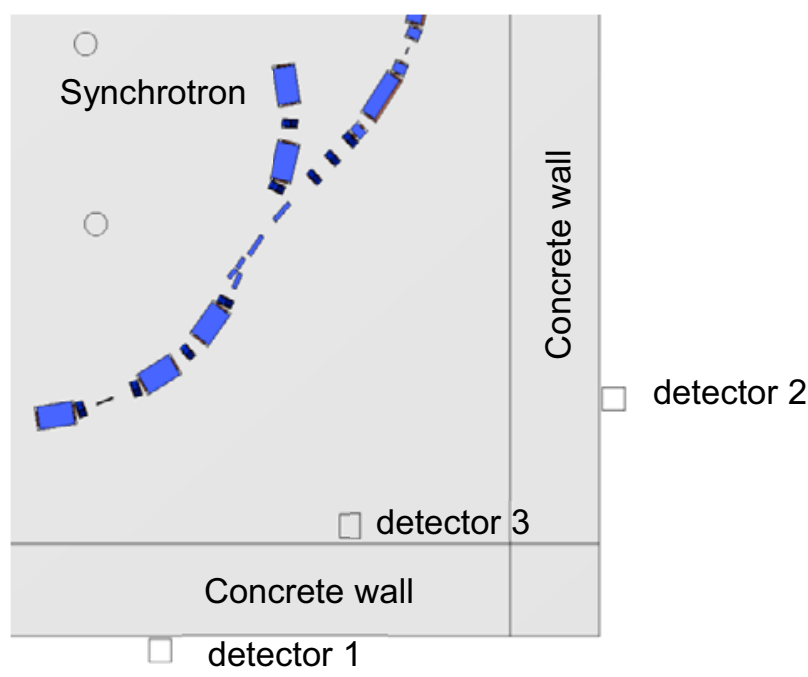

Figure 6. Scoring volumes inside and around the synchrotron hall. 
The total fluence $\Phi$ in the detector volumes consists of the sum of the single fluences $\Phi_{\mathrm{i}}$ created by each of the four loss points:

$$
\frac{d \Phi}{d E}=\sum_{i} w_{i} \frac{d \Phi_{i}}{d E}
$$

with

$$
w_{i}=\frac{\alpha_{i}}{\sum_{i} \alpha_{i}}
$$

where $\mathrm{w}_{\mathrm{i}}$ represents the fracture of the particles loss sum at each loss point where $i=1$ to 4 is the number of the loss point. $\alpha_{i}$ is the number of $800 \mathrm{MeV}$ protons lost annually.

The absolute statistical error, originating from the simulations, was weighted and then propagated as independent error.

Figure 7 to Figure 9 show the spectra originating from particle losses at specific loss points around the synchrotron.

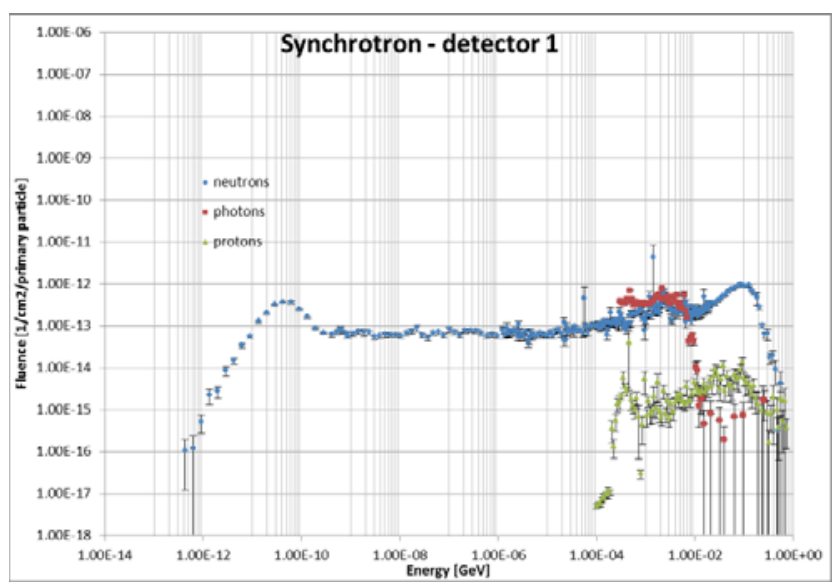

Figure 7. Particle fluence spectra for detector 1

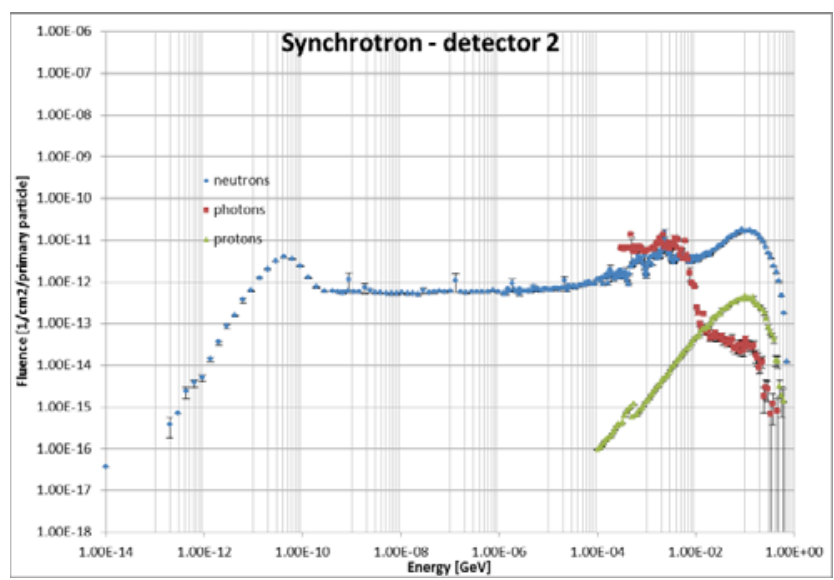

Figure 8. Particle fluence spectra for detector 2

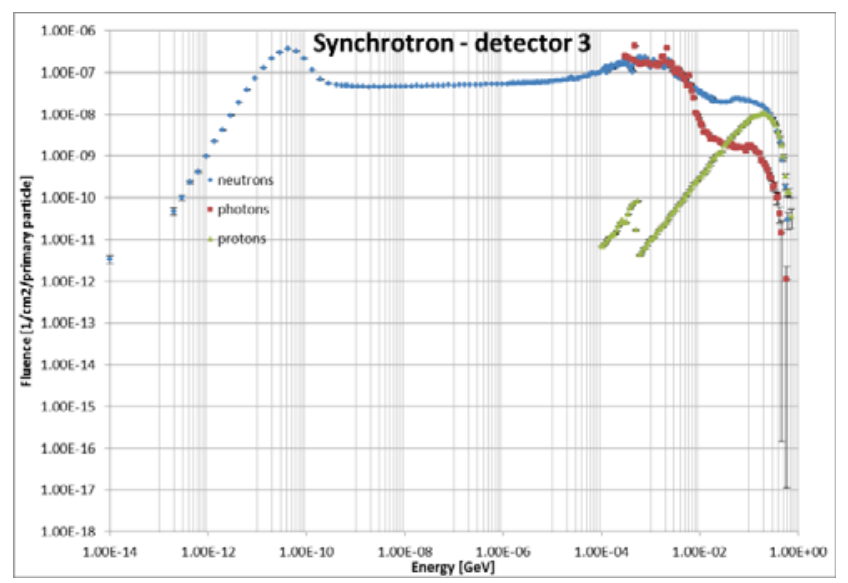

Figure 9. Particle fluence spectra for detector 3

\subsubsection{Contributions to annual ambient dose equivalent values by different particle types}

Table 2 shows the relative ambient dose equivalent contributions by neutrons, photons and protons, which are the main contributors to ambient dose equivalent rates in and around the synchrotron. The values are derived by convolution of the fluence spectra with the respective ambient dose equivalent conversion function by Pelliccioni. ${ }^{6}$ The conversion functions were linearly interpolated in order to be able to perform the convolution.

Table 2. Annual ambient dose equivalent contributions in the

\begin{tabular}{|c|c|c|c|c|c|c|}
\hline \multicolumn{7}{|c|}{ Synchrotron hall } \\
\hline $\begin{array}{l}\text { Ambient } \\
\text { dose } \\
\text { equivalent } \\
\text { rate from }\end{array}$ & 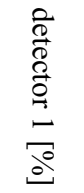 & $\begin{array}{c}+/- \\
{[\%]}\end{array}$ & $\begin{array}{l}\stackrel{2}{0} \\
\stackrel{0}{0} \\
\stackrel{0}{0} \\
\stackrel{0}{0} \\
\stackrel{0}{0} \\
\frac{0}{0}\end{array}$ & $\begin{array}{c}+/- \\
{[\%]}\end{array}$ & $\begin{array}{l}\stackrel{0}{0} \\
\stackrel{0}{0} \\
\stackrel{0}{0} \\
\stackrel{0}{0} \\
\omega \\
\stackrel{0}{0}\end{array}$ & $\begin{array}{l}+/- \\
{[\%]}\end{array}$ \\
\hline Neutrons: & 96 & 0,15 & 91 & 0,08 & 88 & 0,01 \\
\hline Photons: & 1 & 0,11 & 1 & 0,05 & 1 & 0,01 \\
\hline Protons: & 3 & 0,55 & 8 & 0,12 & 11 & 0,09 \\
\hline $\begin{array}{l}\text { Total } \\
\text { dose: } \\
{[\mathrm{mSv} / \mathrm{a}]}\end{array}$ & 0.001 & 0.10 & 0.017 & 0.05 & 211 & 0.01 \\
\hline
\end{tabular}
detectors of the synchrotron hall.

\section{Summary and Conclusion}

Extensive Monte-Carlo simulations were performed and for this purpose a detailed geometry of the MedAustron facility, based on the architectural plans, was developed and implemented in FLUKA simulations. The premise of these simulations is a map of foreseen particle loss points along the accelerator chain. Simplified models of magnets were implemented close to these loss points to consider the shielding effect of the massive iron yokes. The information about the annually lost particles was then used 
as source terms for the FLUKA simulations. The results of the single FLUKA simulations were then converted to annual ambient dose equivalent rates $\left(\mathrm{H}^{*}(10)\right)$ and summed up to build a full annual dose equivalent rate map which shows that the shielding concept of the MedAustron facility is capable of reducing the effect of secondary radiation on humans and the environment below acceptable values, according to the legal limits in Austria. The simulation was an iterative process and input was given to the architect if shielding dimensions were too small or if a dump was needed in specific walls. The architect was then adapting the plans according to the results of the FLUKA simulations.

A more detailed analysis of the radiation field compositions clearly shows that neutrons are the main contributors to ambient dose equivalent rates outside but also inside the shielding walls of MedAustron. Furthermore the neutrons can reach up to primary beam energies with a clear quasielastic peak at around $100 \mathrm{MeV}$. These facts have to be taken into consideration for the design of a radiation monitoring system.

\section{References}

1) (C) Thomas Kästenbauer

2) A. Fassò, A. Ferrari, J. Ranft, and P.R. Sala, FLUKA: a multi-particle transport code, CERN Yellow Report, INFN/TC 05/11, SLAC-R-773, (2005).

3) G. Battistoni, S. Muraro, P.R. Sala, F. Cerutti, A. Ferrari, S. Roesler, A. Fassò, J. Ranft, The FLUKA code: Description and benchmarking, Proceedings of the Hadronic Shower Simulation Workshop 2006, Fermilab 6--8 September 2006, M. Albrow, R. Raja eds., AIP Conf. Proc., 896, pp. 31-49. (2007)

4) http://www.medaustron.at (25.01.2016)

5) C. Theis, K.H. Buchegger, M. Brugger, D. Forkel-Wirth, S. Roesler, H. Vincke: Interactive three dimensional visualization and creation of geometries for Monte Carlo calculations, Nuclear Instruments and Methods in Physics Research A 562, pp. 827-829 (2006).

6) M. Pelliccioni, Overview of Fluence-To-Effective Dose and Fluence-To-Ambient Dose Equivalent Conversion Coefficients for High Energy Radiation calculated using the FLUKA Code, Radiation Protection Dosimetry, Vol. 88, No. 4, pp. 279-297, (2000). 